\title{
Lexikalische Entwicklungen bei Nachfahren nordböhmischer Einwanderer in Rio Grande do Sul - innersprachliche Entwicklungen und deutsch-portugiesischer Sprachkontakt
}

\author{
Sebastian Kürschner ${ }^{1}$ \\ Lehrstuhl für Deutsche Sprachwissenschaft, Katholische Universität Eichstätt-Ingolstadt, Eichstätt, Deutschland
}

Angélica Prediger ${ }^{2}$

Lehrstuhl für Deutsche Sprachwissenschaft, Otto-Friedrich-Universität Bamberg, Bamberg, Deutschland

\begin{abstract}
In diesem Beitrag werden Daten aus einer Studie zur lexikalischen Variation der derzeitigen Einwohner von drei Orten in Rio Grande do Sul untersucht, die durch Einwanderung aus Nordböhmen gekennzeichnet sind. Die Datenerhebung für 40 Wörter (größtenteils Substantive) folgte den Prinzipien der pluridimensionalen Dialektologie. Pro Wort wurden in drei Schritten Varianten (spontane Nennung, weitere vertraute Varianten, von den Interviewern vorgeschlagene Varianten) und Metakommentare erhoben. Mit einer qualitativen Datenanalyse werden Veränderungen und Erweiterungen in der Lexik in Brasilien ermittelt. Die Ergebnisse zeigen, dass komplexe Kontaktsituationen die Entwicklung der Varianten prägen: Varianten der ursprünglichen Dialekte sind selten. Die Daten deuten darauf hin, dass sich häufig westmitteldeutsche und standardsprachliche Varianten durchgesetzt haben. Portugiesische Lehnwörter spielen eine große Rolle, insbesondere, wenn sie Gegenstände von alltäglicher Relevanz bezeichnen, die in der brasilianischen Umgebung neu waren und sind.
\end{abstract}

Schlüsselwörter: Mehrsprachige Gebiete; Deutsch-portugiesischer Sprachkontakt; Lexikalische Veränderung und Erweiterung.

Title: The development of lexical items among descendants of Bohemian immigrants in Rio Grande do Sul, Brazil - linguistic developments within German and German-Portuguese language contact

Abstract: We use data from a questionnaire study on lexical variation in the German of current inhabitants of three places characterized by immigration from Northern Bohemia in Rio Grande do Sul. Data elicitation for 40 words (mostly nouns) followed the principles of Pluridimensional Dialectology, providing the opportunity to include variants from three steps of elicitation per lexeme (spontaneous answers, additional variants the informants were aquainted with, additional answers suggested by the interviewer) and meta comments. The data are used to take a qualitative look at ways in which the lexicon was changed and expanded in Brazil. The results show that complex contact situations shaped the development of the varieties: Variants from the original dialects are rare. The data suggest that they were frequently replaced by West Middle German and Standard variants. Portuguese loan words

\footnotetext{
${ }^{1}$ Professor für deutsche Sprachwissenschaft. Katholische Universität Eichstätt-Ingolstadt. Orcid: https://orcid.org/0000-0001-6364-045X.E-mail: sebastian.kuerschner@ku.de

2 Promotion in Soziolinguistik. Otto-Friedrich-Universität Bamberg. Orcid: https://orcid.org/0000-0001-74366118. E-Mail: angelica.prediger@uni-bamberg.de
} 
play a strong role, especially when they designate items of everyday relevance that were new in the Brazilian surroundings.

Keywords: Multilingual communities; German-Portuguese language contact; Lexical change and expansion.

Título: $O$ desenvolvimento de itens lexicais entre descendentes de imigrantes boêmios no Rio Grande do Sul, Brasil - desenvolvimentos linguísticos entre o alemão e o português em contato

Resumo: Neste artigo, são analisados dados de um estudo de questionário sobre a variação lexical no alemão de descendentes de imigrantes do norte da Boêmia em três localidades no Rio Grande do Sul. A escolha dos dados referentes a 40 palavras (principalmente substantivos) investigadas seguiu os princípios da Dialetologia Pluridimensional, proporcionando a oportunidade de incluir, na análise, variantes de três etapas da entrevista (respostas espontâneas, variantes adicionais que os informantes conheciam, respostas adicionais sugeridas pelo entrevistador) e metacomentários. Foi realizada uma análise qualitativa das maneiras como o léxico foi se modificando e expandindo no Brasil. Os resultados mostram que situações de contato complexas moldaram o desenvolvimento das variedades: as variantes dos dialetos originais são raras. Os dados sugerem que variantes do alemão do oeste da Alemanha e variantes do alemão standard se sobressaíram. As palavras emprestadas do português apresentam um papel importante, especialmente quando designam itens de relevância cotidiana novos no contexto brasileiro.

Palavras-chave: Comunidades multilíngues; Contato alemão-português; Mudança e expansão lexical.

\section{Einleitung}

Die Auswanderung aus Europa nach Süd- und Nordamerika stellt eine zentrale Größe der europäischen Kulturgeschichte und der Herausbildung und Entwicklung der amerikanischen Staaten dar. In Brasilien wurden neue Zuwanderungsgruppen aus Europa v. a. ab dem 19. Jahrhundert angeworben und angezogen (vgl. CUNHA, 2006). Darunter waren auch deutschsprachige Gruppen, die sich ab 1824 im Süden Brasiliens (CUNHA, 2006) ansiedelten und bis heute vor allem für die Bundesstaaten Rio Grande do Sul, Santa Catarina und Paraná prägend sind. Im Rahmen der Einwanderung kamen in Brasilien Menschen aus unterschiedlichen Regionen des deutschsprachigen Raums zusammen (vgl. NAUMANN, 2004, ROSENBERG, 2018), was eine eigene, brasilianische Entwicklung deutschsprachiger Kulturen zur Folge hatte. ${ }^{3}$

Im vorliegenden Aufsatz beschäftigen wir uns mit Daten aus drei Orten im südlichsten Bundesstaat Brasiliens, Rio Grande do Sul (RS), an denen Auswanderung aus Nordböhmen stattgefunden hat. Die Auswanderung fand verstärkt im Zeitraum 1870-1890 statt (FENDRICH,

\footnotetext{
${ }^{3}$ Wir beziehen uns hier in erster Linie auf den sprachlichen, nicht den politischen Raum. Deutsch als Sprache wird in mehreren europäischen Ländern verwendet und ist deshalb nicht nur mit Deutschland verknüpft - in diesem Artikel wird es spezifisch um Böhmen und damit damalig österreichisches Gebiet gehen. Weiterhin war die Zeit der Auswanderung im 19. Jh. insbesondere bei der beginnenden Auswanderung noch durch die Nationenbildung in Europa geprägt, wobei die Nation Deutschland erst im Entstehen begriffen war. Ein deutschsprachiger Kulturraum lässt sich für diese Zeit aber bereits annehmen und mit der sich etablierenden Standardsprache in Verbindung bringen. Auch wenn teilweise eine vereinfachte Ausdrucksmöglichkeit mit „deutsche Einwanderung“ verwendet wird, ist hier also die deutschsprachige Einwanderung aus unterschiedlichen politischen Einheiten gemeint, die gemeinsam durch die Verwendung der deutschen Sprache gekennzeichnet sind.
} 
2016; ${ }^{4}$ PREDIGER, 2019; HABEL, 2017; WILDFEUER, 2017). Einerseits wurde sie durch soziale und politische Entwicklungen im damaligen böhmischen Gebiet angeregt (cf. FLORES, 1983, S. 85-93; HEISLER, 2005, S. 271), andererseits war sie - im Sinne des brasilianischen Interesses an der Einwanderung - mit Aneignungsprozessen von Ländereien in den Taquari- und PardoTälern (CHISTILLINO, 2004) nach dem Landgesetz von 1850 verbunden. Werbung aus Brasilien und positive Berichte bereits Ausgewanderter wirkten als Anziehungsfaktoren, die zahlreiche Menschen bewegten, sich auf die Reise über den Atlantik und einen Neustart in Brasilien einzulassen (HEISLER, 2005). Es kann angenommen werden, dass die Einwander/-innen in Brasilien nach und nach mehrsprachig wurden bzw. ihre bereits aus Europa importierte Mehrsprachigkeit ausbauten und dass die Nachkommen in mehrsprachigen Umfeldern aufwuchsen, und zwar sowohl im Sinne innerer Mehrsprachigkeit (Erwerb weiterer Varietäten des Deutschen) als auch im Sinne äußerer Mehrsprachigkeit (Erwerb weiterer Kontaktsprachen z. B. anderer, benachbarter Einwanderergruppen und des dominanten Portugiesischen) (cf. ALTENHOFEN, 1996; PREDIGER, 2019; HABEL, 2017).

Dass die deutschen Sprachvarietäten in Südbrasilien weiter verwendet wurden, hängt u. a. damit zusammen, dass bis zum 2. Weltkrieg die deutsche Standardsprache in der Literatur, in der Presse, in den Schulen, in der Kirche und in weiteren offiziellen Bereichen noch sehr präsent war. Laut RIEHL (2010, S. 346) wirkt sich eine solch starke Rolle der Standardsprache für den Spracherhalt begünstigend aus. Der ländliche Faktor spielte ebenso eine Rolle für den Spracherhalt, da die deutschsprachigen Gebiete außerhalb der Metropole Porto Alegre erst im Laufe des 20. Jahrhunderts stärker industrialisiert wurden, was stärkeren Kontakt zu anderen deutschen Varietäten und vor allem zum Portugiesischen mit sich brachte. Seit den 40er-Jahren (mit und nach dem Sprachverbot des Estado Novo) drang der Kontakt zum Portugiesischen auch in der Presse und der Literatur und durch die Nutzung der Mehrheitssprache in offiziellen Bereichen, $u$. a. als Schulsprache, immer stärker in das tägliche Leben der Sprachgemeinschaften vor. Portugiesisch entwickelte sich zur einzigen Sprache der Medien und erlangte hohes Prestige. Der fortschreitende Kontakt zur Mehrheitssprache spiegelt sich in der Zunahme an Entlehnungen aus dem Portugiesischen und hybriden Formen im brasilianischen gesprochenen Deutschen.

In diesem Artikel werden die Sprachkontakteffekte in Bezug auf Sprachwandel und -variation berücksichtigt, mit besonderem Fokus auf Neologismen, semantischen Wandel und Entlehnungen. Wir werden uns im Folgenden auf den Bereich der Lexik konzentrieren und uns insbesondere der Frage zuwenden, in welchem Umfang Kontakt zur sich etablierenden Nationalsprache Portugiesisch festzustellen ist und auf welche Art er sich entfaltete. Dazu sollen die Daten aus dem Teil des Interviews zur Lexik (C-Lex) daraufhin untersucht werden, a) welche lexikalischen Einheiten aus den Herkunftsdialekten erhalten blieben, b) welchen Einfluss Kontaktvarietäten wie die deutsche Standard-(Schrift-)Sprache oder das in RS

\footnotetext{
${ }^{4}$ Liste der Aufzeichnungen von Böhmen, die im 19. Jahrhundert durch den Hafen von São Francisco im Bundesstaat Santa Catarina in Brasilien ankamen, verfügbar unter: https://genealogiaboemia.wordpress.com/2016/01/13/lista-de-imigrantes-boemios-list-of-bohemianimmigrants-2/, abgerufen am 11.08.2020.
} 
dominante gesprochene Hunsrückische auf das Lexikon hatten und c) zu welchem Anteil und in welchen semantischen Bereichen Lusitanismen in das Lexikon einflossen und auf welche Art sie in die deutschsprachigen Varietäten integriert wurden.

\section{Daten und soziohistorischer Hintergrund}

Die Daten, die in der vorliegenden Studie verwendet werden, entstammen einer Erhebung aus dem Jahr 2019. Die Erhebung fand im Rahmen einer Studienreise statt, die elf sozio- und kontaktlinguistisch geschulte Studierende und Wissenschaftler/-innen der Katholischen Universität Eichstätt-Ingolstadt nach RS führte. Gemeinsam mit Studierenden und Kolleg/-innen der Universidade Federal do Rio Grande do Sul wurde eine Feldforschung an drei Orten durchgeführt, die für böhmische Einwanderung bekannt sind (vgl. PREDIGER, 2019): Agudo, Imigrante und Venâncio Aires. Hier wurden im Vorfeld der Erhebungen Einzelpersonen und Familien ausfindig gemacht, die zumindest partiell in vorhergehenden Generationen einen böhmischen Einwanderungshintergrund haben. So konnten in Teilen Einzelinterviews, in Teilen Gruppeninterviews durchgeführt werden. Die Interviews fanden sowohl im städtischen als auch im ländlichen Kontext im Umkreis der drei Ortschaften jeweils im häuslichen Umfeld (selten auch in öffentlichen Gebäuden) statt. Alle Interviews wurden weitgehend auf Deutsch geführt, wobei unter den je zwei bis vier Interviewleiter/-innen immer auch Personen waren, die flüssig Portugiesisch sprechen. Alle Gewährspersonen beherrschten neben dem Deutschen auch das Portugiesische.

Wir beschäftigen uns im Folgenden mit den Erhebungen im lexikalischen Bereich. ${ }^{5}$ Die Erhebungsmethodik folgte den Überlegungen der pluridimensionalen Dialektologie (vgl. RADKE \& THUN, 1996; THUN, 2005a, 2009, 2011). Zentrales Merkmal der Methodik ist, dass die Erhebungen unterschiedliche soziale Dimensionen abbilden, die im Falle der lexikalischen Befragung durch die Zusammensetzung der Gewährspersonen reflektiert werden. Die diastratische Dimension etwa wird durch Berücksichtigung von Personen mit höherem bzw. niedrigerem Bildungshintergrund berücksichtigt, die diagenerationelle durch Einbezug von Personen höheren und geringeren Lebensalters und die diatopische durch die drei Erhebungsorte.

Ein weiteres zentrales Merkmal der pluridimensional-dialektologischen Methodik ist die Befragungstechnik, die durch drei Schritte gekennzeichnet ist (cf. THUN, 2005b, 2017 [2005]). Mit dieser Befragungsmethodik soll abgesichert werden, dass nicht nur die Sprachreflexion der Gewährspersonen dokumentiert, sondern auch die ihnen bekannte sprachliche Variation erfasst wird. Im ersten Schritt (Fragen) wird die Variable von der/dem Interviewer/-in eingeführt und um Auskunft zu ihrer Realisierung in der Alltagssprache der Gewährspersonen gebeten, etwa indem nach dem lokalen Wort für ,Rücken' gefragt wird. Im zweiten Schritt (Insistieren) wird nach weiteren Varianten für die Realisierung der Variable gefragt, die den Gewährspersonen bekannt sind. Hier werden etwa andere gebräuchliche

\footnotetext{
${ }^{5}$ Wir danken Gerônimo Loss Bergmann für seine Hilfe bei der Aufbereitung der Daten.
} 
Formen für ,Rücken' (z. B. Buckel, Kreuz) oder phonetische Varianten genannt (etwa Rücken vs. Ricken), die durch die Gewährspersonen in der Sprachproduktion genutzt werden oder innen von anderen Sprecher/-innen am Ort rezeptiv bekannt sind und in den Sinn kommen. Im dritten Schritt schließlich (Suggerieren) wird eine vorbereitete Liste von Varianten herangezogen und von der/dem Interviewer/-in einzeln vorgeschlagen. Die Gewährspersonen teilen bei jeder Variante mit, ob sie ihnen bekannt ist. So werden auch wertvolle metasprachliche Informationen gewonnen. Die Suggerierformen wurden im Vorfeld sorgfältig vorbereitet, indem Varianten aus dem Nordböhmischen, aus gängigen deutschen Varietäten in den Ortschaften und ihren Umgebungen (z. B. Hunsrückisch, Westfälisch) und die portugiesischen Entsprechungen bzw. bekannte, in die Varietäten integrierte Lusitanismen zusammengestellt wurden.

Das Fragebuch lehnt sich insgesamt an das Fragebuch des Projekts Atlas LinguísticoContatual das Minorias Alemãs na Bacia do Prata - Hunsrückisch $(A L M A-H)^{6}$ an, das für die Erhebung hunsrückischer Varietäten konzipiert wurde. Dadurch sollte die Vergleichbarkeit zu den Erhebungen dieses Projekts gesichert werden. Gleichzeitig wurde das Fragebuch speziell mit Blick auf Nachkommen nordböhmischer Einwander/-innen ergänzt, etwa durch die oben beschriebene Erweiterung der Suggerierformen. Insgesamt wurden 40 lexikalische Variablen erhoben, und zwar größtenteils Substantive, die verschiedene semantische Bereiche abbilden: Körper und Kleidung, Ernährung, Wohnhaus, Natur und Landwirtschaft, Verwandtschaft, Sport und Spiel. Die vorliegende Studie ist somit als Untersuchung einer relativ kleinen Stichprobe anzusehen, die erste Einblicke in den lexikalischen Wandel ermöglicht.

Die drei einbezogenen Orte sind im Landesinneren von RS gelegen. Sie sind gemeinsam dadurch gekennzeichnet, dass sie zu den sogenannten ,alten Kolonien' gehören. Die ,alten Kolonien' entstanden in der Umgebung von Porto Alegre, der heutigen Hauptstadt von RS, als erste Siedlungsgebiete deutschsprachiger Einwander/-innen entlang der Täler in Richtung Norden und Westen. Die Nachkommen migrierten zu späteren Zeitpunkten teilweise noch weiter in das Landesinnere, wo dann sog. ,neue Kolonien' entstanden.

Die Ortschaften, die hier einbezogen werden, wurden auf Grundlage einer früheren Datenerhebung bereits in einer Dissertation untersucht (PREDIGER, 2019). Im Rahmen der hier dokumentierten Datenerhebung wurden weitere Gewährspersonen einbezogen, um die Datenbasis zu erweitern. Die Orte wurden ab den 1850er-Jahren durch deutschsprachige Gruppen besiedelt, böhmische Zuwanderung ist ab den 1870er-Jahren dokumentiert (zu böhmischen Familiennamen vgl. PREDIGER, 2019, S. 39-41). Mehrsprachigkeit war in der Region bereits im Vorfeld durch die indigene Bevölkerung, frühen europäischen Zuzug und die Konsequenzen des Sklavenhandels gegeben (LAROQUE et al., 2019; CHRISTILLINO, 2004). Im 19. Jahrhundert kamen neben deutschsprachigen Gruppen noch weitere europäische Gruppen hinzu. Besonders erwähnenswert ist der Zuzug größerer Gruppen aus Italien ab den 1880er-Jahren. Heute sind die Ortschaften durch den Einfluss großer Städte gekennzeichnet

\footnotetext{
${ }^{6}$ Website: https://www.ufrgs.br/projalma/. Abgerufen am 09.10.2020.
} 
(wobei Venâncio Aires selbst ein regionales Zentrum darstellt). Insbesondere jüngere Bewohner/-innen pendeln häufig in umgebende Städte.

\section{Forschungsüberblick und theoretische Vorüberlegungen}

\section{Bisherige Forschung zur lexikalischen Entwicklung in deutschen Varietäten Brasiliens}

Lexikalische Entwicklungen wurden bislang in wenigen Arbeiten dezidiert ins Zentrum der Forschung zu deutschen Varietäten Brasiliens gestellt. Wenn dies der Fall war, so wurde v. a. die Entlehnung aus dem Portugiesischen behandelt. Zu Entlehnungen aus dem Portugiesischen ins gesprochene Hunsrückische im La Plata-Becken hat TAVARES DE BARROS (2019) in seiner Dissertation eine umfangreiche Analyse von mündlichen Daten aus dem bereits oben genannten ALMA-H-Projekt durchgeführt. Einige dieser Entlehnungen finden sich auch in der Sprache der Nachfahren böhmischer Einwanderer wieder, wie sie hier behandelt wird. Ein Beispiel ist die Benennung von Popcorn, z. B. Puffmilje, Pockmilje, Platzmilje. Alle Komposita sind mit einer entlehnten Form für, Mais' gebildet: Milje < pt. milho. Sie stellen als Kombination aus deutschen und portugiesischen Elementen hybride Komposita dar. Die Erstglieder gehen laut dem Verfasser auf die Geräusche zurück, die bei der Zubereitung - beim Platzen des Popcorns - entstehen: „Sie haben als gemeinsames Merkmal die Verwendung von Onomatopoetika der deutschen Sprache (Puff, Platz, Pock) (...) für die lexikalische Komposition, bei denen die Bildung von Hybriden sich anbot. (TAVARES DE BARROS, 2019, S. 124, Übersetzung der Autoren) ${ }^{7}$.

Neben Hybriden zeigt TAVARES DE BARROS (2019) auch lexikalische Varianten aus deutschen Dialekten, z. B. bei Bezeichnungen für die Kartoffel, von denen einige auch bei böhmischen Nachfahren auftreten und in diesem Aufsatz betrachtet werden, vgl. Kartoffel, Grundbirne/-beere, Erdapfel, daneben auch Batate (vgl. Kochbatate, Rosebatate, Weichbatate), das aus pt. batata entlehnt sein könnte. Es ist laut Autor jedoch unsicher, ob es sich um einen Lusitanismus handelt oder ob die Form Patate bereits im deutschsprachigen Repertoire vorhanden war (vgl. TAVARES DE BARROS, 2019, S. 117 mit Rückbezug auf MÜLLER, 1941, S. 557).

Staub (1983) beschreibt pt. Entlehnungen im gesprochenen Hunsrückischen in Santa Cruz do Sul. Neben den hybriden Varianten Brotmilje und Miljebrot ,Maisbrot' beschreibt er auch Komposita, die nur entlehnte Elemente enthalten, vgl. Miljeros ,Maisplantage' mit Ros < pt. roça ,Feld'. Staubs Arbeit zeichnet sich auch durch die Beschreibung lautlicher Integrationsprozesse aus. So beschreibt er z. B. die Integration durch Apokope mit Ersatzdehnung in betonter Silbe, z. B. bei Lagoo < pt. lagoa ,See, Lagune' (Staub, 1983, S. 121). Weiterhin wird die Zentralisierung finaler Vokale zu Schwa als Integrationsmittel beschrieben, vgl. Iskeere < pt. isqueiro ,Anzünder, Feuerzeug' (Staub, 1983, S. 133).

\footnotetext{
${ }^{7}$ Im Original: Los mismos poseen como característica común el uso de onomatopeyas de la lengua alemana (puff, platz, pock) (...) para la composición léxica, donde se oportunizó la formación de hibridismos. (TAVARES DE BARROS, 2019, S. 124)
} 
Einen anderen Ansatz wählt Gärtner (2011), die auf Grundlage von Gesprächen herausarbeitet, wie sich deutsch- und portugiesischsprachige Elemente bei Sprecher/-innen des Bairischen in São Bento do Sul, Santa Catarina, mischen. Neben klassischem CodeSwitching stellt sie fest, dass zahlreiche lexikalische Entlehnungen aus dem Portugiesischen verwendet werden. Dabei arbeitet sie heraus, dass Entlehnungen aus dem Portugiesischen in bestimmten semantischen Bereichen besonders häufig sind: Zahlen, Berufsbezeichnungen und berufliche Fachausdrücke, Verwandtschaftsbezeichnungen, Technik, Schule, Behörden und Institutionen, Begriffe für die spezifisch brasilianische Umgebung und Umwelt. Weiterhin ist laut Gärtner auffällig, dass Interjektionen (z. B. pois é, nossa) und expressive Ausdrücke genauso wie Konjunktionen und Subjunktionen häufig portugiesisch geprägt sind. Daneben stellt sie syntaktische Lehnkonstruktionen fest, bei denen portugiesische Fügungen syntaktisch beibehalten, aber mit deutschem Lexemmaterial ersetzt werden (teuer kosten < pt. custar caro).

Noch relativ wenig systematische Berücksichtigung findet bislang die innersprachliche Variation, die durch Dialektkontakte innerhalb des Deutschen geprägt wurde und wird. Weiterhin wird fast nur auf Entlehnungen fokussiert. Lexikalischen Entwicklungen im deutschen Wortschatz, etwa der Bildung von Neologismen, wird weniger Beachtung geschenkt. Im Folgenden wollen wir die obigen Aspekte aufnehmen und ausgehend von einer kompetenzorientierten Fragebogenstudie Muster der lexikalischen Entwicklung herausarbeiten, wie sie sich bei den böhmischen Nachfahren zeigen.

\section{Theoretische Vorüberlegungen}

Das Lexikon von Sprachen und Varietäten befindet sich in einem stetigen Wandel. Insgesamt gehört das Lexikon zu den Bereichen der Sprache, an denen sich Wandeltendenzen am stärksten ablesen lassen. Nübling et al. (2017, S. 14) entwerfen ein sog. Zwiebelmodell der Sprache, in dem die Kerngrammatik (Phonologie, Morphologie, Syntax) als von äußeren Einflüssen relativ geschützter Teil der Sprache im Kern der metaphorischen Zwiebel liegt. Die Lexik bildet eine der äußeren Schichten der Zwiebel und ist damit anfälliger für außersprachliche Einflüsse, etwa durch gesellschaftlich-kulturellen Wandel, Sprachkontakt oder sprachpolitische Maßnahmen. Noch stärker als die Lexik sind lediglich die Pragmatik und der Text durch äußere Einflüsse veränderlich. Grundlage für die Positionierung der Lexik relativ nah an der außersprachlichen Wirklichkeit ist die Überlegung, dass die Lexik insbesondere zur Referenz auf die gesellschaftliche und räumliche Umgebung dient und sich daher einer sich ständig verändernden Umwelt schnell anpassen muss. Aus diesem Grund müssen etwa Neologismen für neue Objekte geschaffen werden, andere Wörter werden nicht mehr gebraucht. Bedeutungsgleiche Varianten werden auch gesellschaftlich evaluiert, so dass bereits bestehende Lexeme andere ersetzen können oder in ihrer Bedeutung geändert werden (semantischer Wandel).

Bei Varietäten im engen Kontakt mit dominanten Umgebungssprachen, die auf Migration zurückgehen, wie im Fall des Deutschen in Brasilien, lässt sich der Einfluss 
gesellschaftlichen Wandels auf die Sprache im Brennglas beobachten. Einerseits muss die von den Migrant/-innen in einer neuen Umgebung genutzte Sprache den neuen Gegebenheiten gerecht werden. Hierfür werden neue Ausdrücke gebraucht, für die flexible sprachliche Mittel genutzt werden können (Wortbildung, semantischer Wandel). Andererseits etablieren sich bald neue Formen der Mehrsprachigkeit durch Dialekt- und Sprachkontakte, die neue lexikalische Mittel durch Entlehnung zur Verfügung stellen.

Solche Situationen lassen also schnellen lexikalischen Wandel vermuten. So ist auch in Modellen des Sprachkontakts - etwa Thomasons und Kaufmans (1988) Skala der Entlehnungen bei Sprachkontakt mit Spracherhalt - die lexikalische Ebene diejenige, die bereits bei geringem kulturellen Druck der akrolektalen Sprache affiziert wird. Inhaltswörter werden demnach sehr leicht entlehnt, Funktionswörter erst bei gestiegenem kulturellen Druck. Nach Gärtners Darstellung oben wäre bei den bairischen Sprecher/-innen in Brasilien leichter bis leicht gestiegener kultureller Druck anzunehmen, da in erster Linie Inhaltswörter, aber auch Funktionswörter, etwa Subjunktionen, entlehnt werden.

Im Folgenden wollen wir uns mit lexikalischem Wandel bei den Nachfahren böhmischer Einwanderer beschäftigen. Um lexikalischen Wandel zu beschreiben, beziehen wir uns auf allgemein anerkannte Kategorisierungen für Wortbildungsprozesse, semantische Wandelprozesse und die Entlehnung, wie sie etwa in Lehrbüchern dargestellt werden (im Folgenden hauptsächlich an NÜBLING et al. 2017 orientiert, vgl. aber z. B. auch SCHMID 2009, STEDJE 2007). Dafür sollen der Ausbau und weitere Entwicklungen des Lexikons sowohl innerhalb des Deutschen - durch Wortbildung, aber auch Entlehnung aus Nachbardialekten als auch durch Entlehnung aus dem Portugiesischen betrachtet werden. Auf Grundlage der Abfragedaten, die hauptsächlich Substantive betreffen und nur in begrenztem Maße innerdeutsche Wortbildungsprozesse spiegeln, werden wir uns für die innersprachliche Entwicklung des Deutschen insbesondere auch auf lexikalische Wandelprozesse beziehen, die sich in Bedeutungserweiterungen, Bedeutungsverengungen und evtl. Bedeutungsverschiebungen ergeben.

Bedeutungserweiterungen treten dann auf, wenn sich die Extension, also die Menge an Einheiten, auf die ein Begriff angewendet werden kann, erweitert. Gleichzeitig reduziert sich die Zahl an Semen, die zur Beschreibung notwendig sind (Intension). Ein klassisches sprachhistorisches Beispiel hierfür ist Frau, erwachsener weiblicher Mensch' < mhd. vrouwe , adliger erwachsener weiblicher Mensch'. Den gegenteiligen Prozess beschreibt die Bedeutungsverengung, vgl. etwa fahren, sich mit einem Gefährt fortbewegen' < ahd. faran ,sich fortbewegen' (vgl. hierzu und zum Folgenden NÜBLING et al. 2017, S. 144). Bedeutungsverschiebungen liegen dann vor, wenn die Bedeutung eines Wortes sich so weit von einer früheren Bedeutung entfernt, dass sie damit nicht mehr in Zusammenhang gebracht werden kann. So bedeutete witzig zunächst ,klug, vernünftig' und entwickelte seine heutige Bedeutung ,komisch'über die Bedeutung, geistreich' (vgl. noch gewitzt).

Die Einflüsse des Sprachkontakts werden anhand von Entlehnungen herausgearbeitet. Entlehnungen können auf allen Sprachebenen stattfinden. Auf der lexikalischen Ebene wird entlehnt, indem ein fremdes Wort vollständig in die Zielsprache transferiert wird. In einer 
ersten Phase kann das transferierte Lexem mit einem nativen Wort, das als synonym gilt, in Kookkurrenz auftreten. In der weiteren Entwicklung setzt sich evtl. eines der beiden durch, während das andere verdrängt wird.

Durch Entlehnungen können neue Laute oder sogar Lautkombinationen von der Gebersprache in der Zielsprache eingeführt werden. Außerdem können derivationsmorphologische Einheiten transferiert werden und eine eigene Produktivität entwickeln (Lehnwortbildung, vgl. die ins Deutschen entlehnten Derivationsaffixe -er, -ität, ismus, -ieren etc.). Hier können auch hybride Formen eintreten, wenn native Stämme mit einem entlehnten Affix kombiniert werden, vgl. gastieren (vgl. NÜBLING et al. 2017, S. 181).

Die Struktur vieler entlehnter Wörter wird durch Integrationsprozesse der Zielsprache angepasst. Dies kann zu unterschiedlichen Graden passieren. Integrationsprozesse können auf der lautlichen, der graphischen und der morphologischen Ebene beobachtet werden. In unseren Daten ist insbesondere die lautliche Ebene interessant, da weder Flexionsformen der Lexeme noch Schriftsprache sowie kaum Wortbildungsprodukte erhoben wurden.

Die lautliche Integration kann auf prosodischer (etwa Verlagerung des Wortakzents, z. B. zur typisch deutschen Initialbetonung) oder segmentaler Ebene beschrieben werden. Hier spielt insbesondere eine Rolle, dass unbetonte Silben im Deutschen zumeist Reduktionsvokale aufweisen (Schwa), was etwa beim Portugiesischen nicht der Fall ist. Eine lautliche Integration kann so durch vokalischen Wandel geschehen und das Wort den Strukturen deutscher Erbwörter ähnlicher machen (vgl. Milje < milho ,Mais' oben) oder in Kürzung durch Apokope resultieren, vgl. Lagoo < lagoa ,Lagune, See'.

Nicht immer werden Wörter der Gebersprache direkt übernommen. Sprachkontakt macht sich auch dadurch bemerkbar, dass neue Inhalte nach Vorbild der Gebersprache mit nativem Material ausgedrückt werden (semantische Entlehnung, Lehnprägung, vgl. NÜBLING et al. 2017, S. 178). Weiterhin können auch syntaktisch komplexe Einheiten, etwa Phraseologismen, im Sinne von Wort-für-Wort-Übertragungen ins Lexikon eingehen, vgl. teuer kosten oben.

Anhand der skizzierten lexikalischen Entwicklungsmöglichkeiten werden wir uns im Sinne eines methodischen Instrumentariums im Folgenden der Analyse der Befragungsdaten zuwenden. Dabei kann insbesondere auch die den Gewährspersonen bekannte lexikalische Variation einbezogen und danach bewertet werden, in welchem Stadium der Befragung (Spontanantwort, Insistierantwort oder Suggerierform) spezifische Varianten aufgetreten sind. Danach lässt sich zum Beispiel einschätzen, für wie etabliert die Gewährspersonen Lusitanismen im eigenen Sprachgebrauch halten.

\section{Lexikalische Analyse}

Im Folgenden präsentieren wir anhand von vier Beispielwörtern, wie die Variation in den Daten sich gestaltet. Im Anschluss erstellen wir eine systematische Übersicht über die Prozesse, die im Aufbau der Lexik festgestellt werden können, insbesondere mit Blick auf die Erweiterung des Lexikons. Zum Abschluss stellen wir die Nutzung von Lusitanismen im 
Zusammenhang mit den Befragungsschritten und bezogen auf eine semantische Einordnung dar, um sie bezüglich ihrer Etabliertheit einzuordnen.

Beispielhafte Analysen von Einzelwörtern

,Fledermaus'

Am Beispiel ,Fledermaus' lassen sich die vielfältigen Quellen zeigen, aus denen in den untersuchten Varietäten geschöpft wird. Erfragt wurde der Begriff durch die folgende Umschreibung: „Ein schwarzes Tier, dass in der Nacht herumfliegt.“ Tab. 1 bietet zunächst eine Übersicht zur für die Bedeutung ,'Fledermaus' genutzten Lexik.

Tab. 1 - Lexikalische Variation bei ,Fledermaus', pt. morcego



Die Übersicht zeigt zunächst, dass die standarddt. Variante Fledermaus mit lautlicher Variation (z. B. Fladdermaus, Flodermäuser) in den Befragungsorten vorhanden ist. Auch das pt. Lexem morcego wird in den deutschsprachigen Varietäten genutzt, und zwar ohne erkennbare lautliche Integration. An zwei Erhebungsorten wird zudem eine dialektale Variante genannt, Speckmaus. Diese Variante ist in mitteldt. Varietäten bekannt (vgl. etwa PfW mit Karte unter dem Eintrag Fledermaus, auch DtW) und hat sich somit wahrscheinlich über die hunsrückischen Kontaktvarietäten etabliert. Mit Nachtschatten und Nachteule, den beiden weiteren lexikalischen Belegen, sind wohl Eulen gemeint, die versehentlich aufgrund ihrer Nachtaktivität bei den Gewährspersonen in den semantischen Rahmen von Fledermäusen gelangt sind.

Die Tabelle zeigt, dass zu einigen der Varianten auch metasprachliche Kommentare zur Verfügung stehen. So wird deutlich, dass Speckmaus in Teilen eher als Spitzname (für 
Menschen) genutzt wird, bei dem die Tiermetaphorik der Fledermaus oder die Anspielung auf den Speck noch eine Rolle spielt. Weiter geht eine Gewährsperson aus Imigrante davon aus, dass Speckmaus in der Gegend kaum gebräuchlich sei, eine andere davon, dass dies die häufigere Variante in der Gegend sei. So zeigt sich, dass am Ort hohe inter- und intraindividuelle Variation vorliegt.

Mit Bezug auf die Untersuchungsorte lässt sich feststellen, dass die Variante Fledermaus in Agudo die einzig mögliche Variante darstellt. Die dialektale Variante Speckmaus wird hier auch in der Suggerierphase abgelehnt. Auch die pt. Variante wird nicht als Teil der Lexik in den deutschen Varietäten angesehen. Im Gegensatz zu den anderen Orten kann in Agudo kaum Variation festgestellt werden (lediglich Flattermäus wird als Suggeriervariante in einem Interview anerkannt). An den anderen Orten werden dagegen in den unterschiedlichen Interviews alle drei Varianten bereits als spontane Antworten genannt.

,Grillen', pt. Churrasco

Die typisch brasilianische (und iberische) Art, Fleisch am Spieß zu grillen (Churrasco), wird mit unterschiedlichen pt. und dt. lexikalischen Einheiten ausgedrückt. Zur Umschreibung wurde in der Befragung „Fleisch auf einem Spetto “verwendet (pt. espeto, ,Spieß”). Tab. 2 zeigt die vorgefundene Varianz.

Tab. 2 - Lexikalische Variation bei ,Grillen am Spieß', pt. churrasco

\begin{tabular}{lll}
\hline Churrasco & Agu, VenA, Imig & \\
\hline Schurraske & VenA & \\
\hline Steckefleisch & Agu, VenA, Imig & $\begin{array}{l}\text { VenA: "das haben die Eltern immer gesagt", } \\
\text { „früher“ }\end{array}$ \\
\hline Steckelfleisch & Agu, VenA & \\
\hline Spießbrat(e)n & Agu, VenA, Imig & \\
\hline Spießbrout(e)n & VenA, Imig & VenA: „Böhmisch“ \\
\hline Spießbrot(e)n & VenA, Imig & Imig: „Hunsrück“ \\
\hline Spießfleisch & VenA, Imig & \\
\hline Spießbrotefleisch & VenA & \\
\hline Bratefleisch & Imig & \\
\hline Fleischbraten & Imig & Imig: „aber nicht auf Spieß, sondern im Topf \\
& & oder Ofen“ \\
\hline Spetto, Spette & Imig & \\
\hline
\end{tabular}

Quelle: die Autoren.

Das pt. churrasco hat sich an allen Orten etabliert. In Venâncio Aires tritt hier auch eine lautlich integrierte Variante Schurraske auf, bei der der finale Vokal zu Schwa reduziert wurde. Daneben zeigen die Daten verschiedene deutschsprachige Umschreibungen: Steckefleisch/Steckelfleisch, Varianten von Spießbraten sowie Bratefleisch/Fleischbraten 
(diese beschränkt auf Imigrante), daneben Spetto/Spette, das als integrierte Variante (anlautend $/ \mathrm{J} /$, reduzierter Auslaut) ein Lexem der Frage wiederholend aufnimmt. Metonymisch wird hier der ,Spieß' (pt. espeto) als Bezeichnung für den Prozess und die resultierenden Gerichte des Grillens genutzt. Die Variante Grillen, die erst im 20. Jh. aus dem Engl. ins Dt. entlehnt wurde, spielt keine Rolle.

Die metasprachlichen Kommentare zeigen, dass die Varianten teilweise mit bestimmten Nutzungskontexten verbunden werden. So wird die Variante Steckefleisch in Venâncio Aires mit der Sprache von "früher" und der Elterngeneration in Verbindung gebracht - ein Zeichen, dass sich der Lusitanismus Churrasco, der hier bei den Spontanantworten dominiert, in den jüngeren Generationen durchsetzt. Lautliche Variation bei Spießbraten wird mit spezifischen Dialekten in Verbindung gebracht - ein velarisiertes $a$ in Spießbroten in Imigrante etwa mit Hunsrückisch, die diphthongierte Form Spießbrouten in Venâncio Aires mit Böhmisch.

Mit Steckefleisch liegt eine spezifisch brasilianische Neuprägung vor, ein Kompositum, das auf deutschem Material aufbaut. Das Determinandum Fleisch wird hier durch das Determinans Stecke, das auf das Verb stecken oder auf das Substantiv Stecken (mit zumindest im Hunsrückischen verbreiteter $n$-Apokope) zurückgeht, näher bestimmt. Hier wurde also für eine in der europäischen Herkunftsregion wenig verbreitete (oder zumindest wenig charakteristische) Zubereitungsart durch Wortbildung ein Neuwort kreiert, um das in der neuen Umgebung relevante Konzept zu bezeichnen. Daneben wurde das bereits bestehende Kompositum Spießbraten in der Bedeutung eingegrenzt (Bedeutungsverengung). In der Herkunftsküche steht der Begriff für jegliche Form von Braten am Spieß (also auch etwa im Ofen gebackener Braten), in der brasilianischen Region dann spezifisch für gegrilltes Fleisch.

Mit Bezug auf die Erhebungsorte fällt auf, dass die Variante Spießbraten in Agudo nicht als spontane Antwort auftritt, sondern erst in der Insistierphase (bei nur einer Person). Steckefleisch ist neben Churrasco in allen Gesprächen bekannt, während Spießbraten bei einigen Interviewgruppen selbst in der Suggerierphase als unbekannt gekennzeichnet wird.

\section{,Rasieren“}

Da fast nur Substantive erfragt wurden, wird in diesem und dem folgenden Beispiel kurz auf ein Verb und ein Adjektiv eingegangen. Für rasieren - eine im Dt. fest etablierte und integrierte Entlehnung aus dem Französischen - wird das ursprüngliche Verb neben Varianten von barbieren und Umschreibungen verwendet, wie Tab. 3 zeigt. Erfragt wurde die Variable mit folgendem Ergänzungssatz: „Wenn der Bart lang ist, muss man inn ...“.

Tab. 3 - Lexikalische Variation bei rasieren, pt. barbear-se, fazer a barba, tirar a barba

\begin{tabular}{lll}
\hline (sich) rasiere(n) & Imig, VenA, Agu & \\
\hline abrasiere & Imig & \\
\hline der Bart / & Imig, VenA, Agu & VenA: zu Bort "das ist Laschoodisch"; Agu: \\
Bort mache(n) & & zu Bort mache "das ist mehr Sachsisch" \\
\hline
\end{tabular}




\begin{tabular}{lll}
\hline der Bart abmache & Imig, VenA, Agu & \\
\hline sich den Bart machen & Imig & \\
\hline Gillett abmache & VenA & Imig: „schon gehört, aber selten“, \\
\hline (sich) balbiere( $n$ ) & Imig, VenA & "balbieren vom Brasilianischen eingefügt“ \\
\hline (sich) balwiere & Imig, VenA & VenA: "die Älteren haben das gesagt" \\
\hline barbiere & Imig & \\
\hline abschneide( $n)$ & Imig, VenA, Agu & \\
\hline glattmache & Imig & \\
\hline
\end{tabular}

Quelle: die Autoren.

Neben rasieren spielt vor allem die Variante balbieren eine Rolle. Diese lautliche Variante zu barbieren mit $-I$ geht auf Dissimilation zurück (vgl. DWDS). Sie ist in den westmitteldeutschen (wmd.) Ausgangsdialekten bereits verbreitet (vgl. PfW) und wurde so wohl über das Hunsrückische nach Brasilien transportiert. Die Variante balbieren/barbieren wurde in der deutschen Standardsprache durch rasieren weitgehend abgelöst und wird heute kaum mehr genutzt, während sie in Brasilien durch die portugiesische Form barbear-se eine Verstärkung erfahren haben könnte. In Agudo wird diese Variante nicht genutzt - wiederum findet sich hier die mit der Standardsprache übereinstimmende Variante rasieren neben den im Folgenden besprochenen Lehnübersetzungen.

Interessant sind auch die Varianten zu der/den Bart (ab)mache, die in dieser Form weder in der deutschen Standardsprache noch im Ursprungsdialekt auftreten. Sie lassen sich als Lehnübersetzungen zum portugiesischen phraseologischen Verb fazer a barba bzw. tirar a barba interpretieren. Es handelt sich also um syntaktische Übersetzungen mit deutschem lexikalischen Material. Eine weitere Variante zu dieser Lehnübersetzung ist die mit Gillett abmache. Hier wird die Rasierermarke Gillette metonymisch verwendet und in die syntaktische Form eingefügt. Weitere Ergänzungen des Ausgangssatzes sind abschneiden und glattmachen.

Rasieren und Bart machen sind die Varianten, die unter den Spontanantworten unabhängig vom Erhebungsort am stärksten verbreitet sind. Barbieren/balbieren tritt nur selten als Spontanantwort auf und wird, insbesondere in der Form mit - , nicht durch alle Gruppen als verbreitete Form akzeptiert. Die metasprachlichen Kommentare geben darüber Auskunft, dass die Form z. T. als veraltet empfunden und als portugiesische Entlehnung interpretiert wird. Dies ist einerseits auf die Übereinstimmung bzw. Ähnlichkeit mit dem pt. Verbstamm barb- zurückzuführen, andererseits aber auch auf das Derivationssuffix -ier, das im brasilianischen Kontext häufig zur Integration portugiesischer Verben verwendet wird (vgl. gravieren für ,aufnehmen' aus gravar) - die Häufigkeit des Verfahrens lässt Verben auf -ieren als Lusitanismen wirken, obwohl bereits zahlreiche ieren-Verben aus dem europäischen Deutschen importiert wurden. Weitere metasprachliche Kommentare zeigen, dass lautliche Varianten (Bort mit velarisiertem a) regional (,Laschodisch“ mit Bezug auf die Stadt Lajeado) oder mit Bezug auf den Herkunftsdialekt interpretiert werden („Sachsisch“). 
,Barfuß'

Als Adjektiv soll schließlich die lexikalische Variation in den Antworten zu folgendem Ergänzungssatz vorgestellt werden: „Wenn man nichts an den Füßen hat, dann ist man ...“ Tab. 4 zeigt die lexikalischen Varianten.

Tab. 4 - Lexikalische Variation bei ,barfuß', pt. pé-descalco

\begin{tabular}{lll}
\hline blossfiessich, bloosfüß & Imig & \\
\hline $\begin{array}{l}\text { balfusich } \\
\text { barfüsich, barfusich, } \\
\text { barfiesich, borfusich, } \\
\text { borfisich }\end{array}$ & Agu, VenA, Imig & $\begin{array}{l}\text { VenA: zu borfusich „Lajeado“, „der geht } \\
\text { barfisich, der ist barfusich“ }\end{array}$ \\
\hline barfuß & Agu, Imig & \\
\hline barfußbeinich & Imig & VenA: „Böhmisch“ \\
\hline borbsfiesich & VenA & Imig: zu barbs, „Die Urgroßmutter hat das \\
\hline barbs, borbs & Agu, VenA, Imig & $\begin{array}{l}\text { immer gesagt", zu borbs „, borps am Kopf, } \\
\text { Haare einwickeln“; VenA: zu beiden } \\
\end{array}$ \\
& "Böhmisch“ \\
\hline ohne Schlappen & Agu & \\
\hline
\end{tabular}
Quelle: die Autoren.

Neben der mit dem Standarddeutschen übereinstimmenden Variante barfuß zeigen sich (im europäischen Deutschen mögliche, aber weniger geläufige) Varianten mit dem adjektivischen Derivationssuffix -ig (hier zur Darstellung der frikativischen Realisierung als ich dargestellt). Das Derivat ist im Hunsrückischen verbreitet und bereits in den Herkunftsdialekten vorhanden (vgl. PfW). Variiert wird auch zwischen der unumgelauteten (barfußig) und der umgelauteten (barfüßig bzw. mit Entrundung barfießig) Version des Stammvokals. Daneben finden sich Umschreibungen (barfußbeinich, ohne Schlappen, bloßfießich). Bemerkenswert ist die Variante barbs/borbs (dazu auch das Kompositum borbsfießich). Einfluss des Portugiesischen lässt sich bei dieser Variable nicht eindeutig erkennen, wenn auch die Umschreibung ohne Schlappen mit der phraseologischen Einheit pt. sem chinelo im Sinne einer syntaktischen Lehnübersetzung in Verbindung stehen könnte.

In den Metakommentaren wird bei der lautlichen Variation von bar-wieder die Zuordnung von velarisiertem a zu Lajeado vorgenommen und damit zu einer als durch das Hunsrückische geprägt empfundenen Stadt. Die Variante barbs/borbs wird kaum als Spontanantwort geliefert, jedoch teilweise als Insistierform. Es handelt sich um eine lexikalische Variante, die bei den Suggerierformen als Kennform des obersächsischschlesischen Übergangsdialekts berücksichtigt wurde, der das nordböhmische Auswanderungsgebiet charakterisiert. Bei den Suggerierformen wird barbs/borbs nur in 
wenigen Interviews erkannt und dann teilweise mit metasprachlichen Kommentaren belegt, die es auf die Sprache der Älteren einschränken und einmal spezifisch dem böhmischen Dialekt zuordnen. Dies ist einer der seltenen Fälle, in denen die nordböhmische Varietät sich bis heute niederschlägt, während hunsrückische und standardnahe Varietäten den nordböhmischen Dialekt sonst weitgehend ersetzt haben (vgl. PREDIGER, 2019).

Die Detailbetrachtung der Beispielwörter konnte zeigen, dass die lexikalische Variation durch sowohl außersprachliche (Kontakt zum Portugiesischen) als auch innersprachliche Variation (Dialekte und Standardsprache sowie stilistische Variation des Deutschen) geprägt ist. Die hohe Variation zeigt außerdem, dass das Deutsche in Brasilien bis heute keineswegs einheitlich ist, sondern durch die Sprach- und Varietätenkontakte ortsspezifischer Entwicklungen geprägt ist. Die portugiesische Kontaktsprache und hunsrückische Varietäten spielen dabei eine ausgeprägt dominante Rolle, aber insbesondere Insistier- und Suggerierformen lassen auch den Einfluss weiterer Varietäten erkennen. Für die neue brasilianische Umgebung wurden mit Hilfe der Wortbildung auch neue Wörter auf Grundlage der deutschen Varietäten geschaffen (vgl. Steckefleisch) oder bestehende Wörter in ihrer Bedeutung an neue Kontexte angepasst (vgl. die Bedeutungsverengung bei Spießbraten).

Die Metakommentare zeigen, dass Varianten kontextualisiert interpretiert werden. So wird einerseits eine Verbindung der Varianten zum Alter der Sprecher/-innen und somit ihrer Aktualität hergestellt - wenn Varianten nur noch von den Ältesten verwendet werden oder Verstorbenen zugesprochen werden, heißt das, dass sie von der jüngeren Sprachgemeinschaft nicht mehr als gebräuchlich angesehen werden. Andererseits werden Varianten von den Gewährspersonen mit Bezug auf geographische Variation und die Ausgangsdialekte der Vorfahren interpretiert. Offensichtlich ist also ein Bewusstsein für eine dialektale Vielfalt des Deutschen in Brasilien vorhanden und wird teilweise als geographische Variation im eigenen Land („Laschoodisch“) eingeordnet, teilweise auf Variation aufgrund der ursprünglichen und in Brasilien weiterentwickelten Herkunftsdialekte bezogen („Hunsrückisch“, „Böhmisch“).

\section{Analyse der lexikalischen Entwicklungen in den Belegdaten}

Nach diesen Einzelwortanalysen sollen im Folgenden die lexikalischen Entwicklungen unter Berücksichtigung aller Abfragedaten eingeordnet werden. Dabei werden zunächst innersprachliche Prozesse, im Anschluss die Integration von Lusitanismen behandelt.

Innersprachliche Prozesse: Lexikalische Entwicklungen bei Wörtern aus dem Deutschen

1 Neologismen

Wie oben bereits bei Steckefleisch behandelt, zeigen unsere Daten einige Neologismen, die im brasilianischen Umfeld entstanden sind, um dortige Lebensumstände zu benennen. Wir behandeln hier Komposita, die auf Grundlage deutscher Ausgangswörter 
entstanden sind. Unten behandeln wir dann auch hybride Komposita unter Einbezug von Lusitanismen.

Solche Neologismen, die fast nur als Komposita auftreten, sind in den Daten relativ selten. Sie sind unter anderem durch (in den Daten nicht erfragte) Wörter wie Keesboom „Käsebaum“ bekannt, das etwa eine aus Europa nicht bekannte Baumart bezeichnet (pt. umbu, vgl. Altenhofen 2016, S. 118). Einige Komposita, die sich in Brasilien bewahrt haben, stammen bereits aus den Ausgangsdialekten und sind etwa im PfW dokumentiert, vgl. Riechseif ,wohlriechende Seife', Fixfeuer, Fixholz, Streichfeuer ,Streichholz'. Sie spiegeln technische Neuerungen, die aber bereits im Emigrationsgebiet lexikalisiert wurden und somit keine brasilianischen Spezifika darstellen. In diesen Wortfeldbereichen wurden jedoch weitere Prägungen zur Bezeichnung von Seife verzeichnet, etwa Wasserseif oder Sodaseif. Beim ,Streichholz' fällt die Bezeichnung Feuerding ins Auge, die eine relativ allgemeine Umschreibung darstellt, bei den hier untersuchten Gruppen aber kaum Verwendung findet. Daneben wird als Derivat Zünder als Spontanbeleg verwendet, vermutlich abgeleitet von Zündholz, das als Kompositum bei den Informant/-innen allerdings keine Rolle spielt.

\section{Bedeutungsverengung}

Oben wurde bereits die Bedeutungsverengung bei Spießbraten behandelt. Ein weiteres Belegwort mit Bedeutungsverengung ist bei einem Teil der Gewährspersonen Rücken. Während einige Personen das Wort in der allgemeinen Bedeutung als hinterer Teil des Oberkörpers verwenden, beschreiben andere die Bedeutung eingeschränkt auf den unteren Teil des Rückens. Dies lässt sich in Zusammenhang mit einer allgemeinen Verbreitung der Bezeichnungen Kreuz und Buckel für den ,Rücken'stellen, die semantische Differenzierung der Bezeichnungen zulässt.

Ein weiteres Beispiel besteht bei Kugel, das sich teilweise allgemein für ,(Spiel-)Ball‘ durchgesetzt hat. Als stilistische Variante ist die Nutzung von Kugel auch etwa aus der Sprache des deutschen Sportjournalismus in der Berichterstattung zu Ballsportarten bekannt, in den hier behandelten Varietäten wird das Wort aber teilweise verallgemeinernd insbesondere für den Fußball genutzt. So entstehen auch Komposita wie Fußkuchel, Gummikugel, Lederkuchel. Daneben werden aber auch die Varianten Ball und spezifischer Fußball verwendet.

\section{Bedeutungserweiterung}

Während die Bezeichnung Schmier bereits in den wmd. Ausgangsdialekten für Brotbestriche süßer (Süßschmier im Sinne von Marmeladen aller Art) und saurer Art (Kässchmier) sowie entsprechende belegte Brote verwendet wurde (vgl. etwa im PfW) und so auch heute in Brasilien Verwendung findet, wurde beim Wort Sirup eine Verallgemeinerung der Bedeutung im Sinne von ,Marmelade' durchlaufen. Das Wort Gewitter/Sommergewitter wurde von einigen Gewährspersonen in der verallgemeinerten Bedeutung auf ,Sturm' bezogen. 


\section{Bedeutungsverschiebung}

Gefragt nach dem Fischteich, werden die Bezeichnungen Sump(f), Flusse und Bach genannt. Der Sumpf bezieht sich eigentlich auf feuchtes Land und erfährt hier eine Bedeutungsverschiebung zu einer Wasserstelle, die zum Heranziehen von Fischen geeignet ist. Die Variante ist nur in Imigrante verbreitet und wird dort als Spontanbeleg genutzt. Bei Flusse und Bach handelt es sich um Bedeutungsverschiebungen bei Lexemen, die ursprünglich fließende Gewässer bezeichneten. Hier könnten fließarme Abgrenzungen solcher Fließgewässer, etwa Auen oder Buchten, zugrunde liegen, die eine Fischzucht zulassen.

5 Integration von Lexemen aus anderen Varietäten

Die bisherigen Betrachtungen zeigen bereits, dass sich die Lexik in den deutschsprachigen Varietäten der innersprachlichen Variation bedient, insbesondere indem die Standard-(Schrift-)Sprache Einfluss nimmt und hunsrückische Varietäten deutlich als lexikalische Leitvarietäten auf mündlicher Ebene dienen (vgl. lexikalische Varianten aus westmitteldeutschen Varietäten wie Persching ,Pfirsich', Gommer ,Gurke', Hosensack/-säckel „Hosentasche', Grundbeere ,Kartoffel', Hinkel ,Huhn', zackern ,pflügen' etc.). Dass auch andere Einwanderervarietäten eine Rolle spielen, zeigt sich einerseits an der bereits oben behandelten Bewahrung weniger lexikalischer „Reste“ der nordböhmischen dialektalen Varietät (in unseren Daten nur oben behandeltes borbs , barfuß'), andererseits daran, dass lexikalische Varianten wie Bucks ,Hose' evtl. durch niederdeutsche Varietäten verstärkt werden könnten, selbst wenn sie auch in wmd. Varietäten vorhanden sind (vgl. PfW). Weitere Varietäten spielen, ebenso wie andere Kontaktsprachen als das Portugiesische, aber in unseren Daten eine deutlich untergeordnete Rolle.

\section{Außersprachliche Prozesse: Die Integration von Lusitanismen}

\section{Lexikalische Entlehnungen}

Lexikalische Entlehnungen bilden den häufigsten Fall sprachlicher Einheiten, an denen sich Einfluss des Portugiesischen ablesen lässt. Relativ selten werden Wörter dabei schlicht auf Portugiesisch verwendet - eine Tatsache, die in Zusammenhang mit der Art der Daten steht, also der lexikalischen Befragung. Aus der Untersuchung freierer Gespräche ergibt sich hingegen, dass portugiesische Wörter häufig unverändert übernommen werden, insbesondere wenn entsprechendes Vokabular in der deutschen Varietät fehlt (vgl. Gärtner 2011) - alle Sprecher/-innen in unseren Erhebung beherrschen das Portugiesische fließend, so dass eine Nutzung des portugiesischen Wortschatzes bis hin zum Code-Switching jederzeit flexibel möglich ist. In unseren Befragungsdaten finden sich unveränderte Übernahmen als Spontan- oder Insistierbelege etwa bei churrasco ,Grillen', geléia ,Marmelade', isqueiro ,Feuerzeug', piso ,Fußboden', pêssego ,Pfirsich', etc. 
Zahlreiche Lusitanismen durchlaufen dagegen eine lautliche Integration. Insbesondere findet diese Integration durch zwei Prozesse statt, nämlich die Reduktion unbetonter Vokale zu Schwa und die Apokope. Beispiele für die Vokalreduktion bilden Milje, Milgen < milho 'Mais', Fosfore < fósforo ,Streichholz', Belmude < bermuda ,Bermudashorts' - hier interessanterweise im Kontrast zum europäischen Deutschen, in dem Bermuda klar in Fremdwortstruktur erhalten bleibt -, Funde < funda ,Schleuder', Schurraske < churrasco 'Grillen', Aranje < aranha ,Spinne', hier metaphorisch für einen kleinen Ochsenwagen gebraucht, Isqueere < isqueiro ,Streichholz', Lage < lago ,See', etc.

Mit Apokope des finalen Vokals treten z. B. folgende Wörter auf: Sabonett < sabonete ,Seife', Pipock < pipoca ,Popcorn', Batat < batata ,(Süß-)Kartoffel', Kamisett < camiseta ,TShirt', Kamisol < camisola ,Hemd', Scharrett < charrete ,kleiner Wagen', Tank < tanque ,Teich', etc. Auch konsonantische Apokope kommt vor, vgl. Silage < silagem ,Silage'. Das finale -e wird teilweise nicht als Schwa, sondern als gehobener Vokal /i/ ausgesprochen, was den portugiesischen Einfluss aufzeigt. Bei manchen Wörtern werden auch ganze Silben apokopiert, vgl. Fosfo < fósforo ,Streichholz', oder die Apokope finalen Vokals wird mit Vokalreduktion kombiniert, vgl. Fosfer, ebenfalls < fósforo.

Weitere lautliche Integration lässt sich feststellen, wenn portugiesisches /3/ durch das stimmlose Gegenstück /J/ ersetzt wird, vgl. Scheleja / Schieleja < geléia ,Marmelade', Barasching < barragem ,Staudamm/Wehr' sowie Schurraske ,Grillen' (s. o.).

Bei Wörtern mit sehr ähnlicher Lautung im Deutschen und Portugiesischen ist der Lehnwortcharakter weniger klar zu erkennen. Gabel etwa wird im wmd. Dialekt häufig als Gawel realisiert (PfW), wodurch bereits eine frikativische Realisierung des anlautenden labialen Konsonanten der zweiten Silbe wie in port. garfo gegeben ist. In unseren Daten finden wir häufig die Realisierung mit $/ r /$, also als Garfel, die somit sowohl nah an der Ausgangsstruktur im Dialekt liegt als auch am Portugiesischen.

\section{Lehnwortbildungen und weitere Derivation}

Im einbezogenen Material finden sich zwar viele lexikalische Entlehnungen (s. o.), jedoch nur selten solche, die mit Mitteln der Derivation ins Deutsche integriert wurden. Dies liegt v. a. an der Zusammenstellung des Materials, das sehr an Substantiven ausgerichtet ist. Im verbalen Bereich lässt sich die Integration z. B. häufig mit Hilfe des Suffixes -ieren nachvollziehen (in unserem Material nur bei balbieren/balwieren zu erkennen, das zudem in dieser Form vermutlich bereits aus dem europäischen Deutschen nach Brasilien gelangt ist, vgl. die Ausführungen oben).

Als Derivat findet sich ein Beispiel für Diminutivbildung, indem für ,Sohn' Gurichen und Gorichen < guri ,Junge' genannt werden. Hier liegt eine lexikalische Entlehnung beim Stamm zugrunde, die mit dem deutschen Diminutivsuffix versehen wird. Interessanterweise wird guri in unseren Daten nur mit Derivationssuffix verwendet, was einen Lexikalisierungsprozess anzeigen könnte, bei dem die Differenzierung zwischen den Bedeutungen ,Sohn' und ,Junge' durch das Diminutionssuffix herbeigeführt wird. 
3 Hybride Komposita

Relativ häufig finden sich Komposita, in denen Lusitanismen mit nativen deutschen Wörtern kombiniert werden. Dies geschieht einerseits bei Komposita mit einem Lusitanismus als Zweitglied, also in den vorliegenden Determinativkomposita als Determinandum, vgl. Futtermilje ,Futtermai', Puffmilje, Puffmülgen, Platzmilje, Buckmilje, Bock(s)milje ,Popcorn', Fischassude ,Fischteich', Sießbatat ,Süßkartoffel'. Andererseits zeigen sich auch Komposita, in denen der Lusitanismus das Determinans bildet, vgl. Miljekenne ,Maiskorn', Fosfeier, Fosfeuer 'Streichholz', Fosfeierschachtel ,Streichholzschachtel'. Dabei tritt auch weitere lautliche Reduktion zutage, vgl. das Erstglied Fos- < fósforo, das auf eine Silbe gekürzt auftritt. Interessant ist das hybride Kompositum Fundenlader, das sich aus Funde < pt. funda ,Schleuder' und Lader ,Leder' zusammensetzt, wobei sich das a als typisches Merkmal für die obersächsisch-schlesischen Dialekte des nordböhmischen Auswanderungsgebiets zeigt - hier also ein lautlicher Beleg für ein bewahrtes nordböhmisches Dialektmerkmal (vgl. PREDIGER \& KÜRSCHNER, i. Dr.).

\section{Syntaktische Lehnprägungen}

Bereits oben (,Rasieren') wurde die syntaktische Lehnprägung der Bart / Bort mache(n), der Bart abmache, sich den Bart machen behandelt, die das einzige Beispiel dieser Art in unseren Daten darstellt (zu ohne Schlappen vgl. die Ausführungen oben unter ,Barfuß'). Die Kategorie wird deshalb der Vollständigkeit halber erwähnt, aber nicht weiter ausgeführt.

\section{Status und semantische Bereiche nativer deutscher Lexik und der Lusitanismen}

Wenn Lusitanismen bereits als Spontanantwort gegeben werden, ist damit zu rechnen, dass sie im allgemeinen Sprachgebrauch der Gewährspersonen fest verankert sind. Hier fällt auf, dass Körperteile und Kleidung sowie zentrale Bereiche des bäuerlichen und handwerklichen Lebens, wie sie bereits in Europa etabliert waren, weitgehend mit nativer Lexik benannt werden, vgl. Rücken/Kreuz/Buckel, Mund, Hemd, Hose, Teller, Nagel, Boden, Fenster, Wagen, zackern ,pflügen'. Hierzu gehören auch enge Verwandtschaftsbezeichnungen, vgl. Mädchen ,Tochter', Bub/Jung 'Sohn', Schwiechermutter/Mitmutter 'Schwiegermutter', etablierte Nahrungsmittel, vgl. Schmier 'Marmelade' bzw. ,fetthaltige Brotschmiere', Gurke/Gommer, Kartoffel und Tiere, vgl. Huhn/Hinkel, Pferd/Gaul. Die dialektalen Formen zeigen nochmals, dass die böhmischen Einwander/-innen und ihre Nachfahren zahlreiche dialektale Formen selbst im engen Alltagsbereich aus dem Wmd. übernommen haben. Sprachliche Ausgleichsprozesse sind also insbesondere innerhalb deutscher Varietäten nachvollziehbar.

Lusitanismen findet man besonders in den Alltagsbereichen bereits als Spontanantwort, bei denen die Umgebung eine Rolle spielt, etwa bei Bezeichnungen für den Fischteich (Assude < pt. açude, Lagoo < pt. lagoa), der in der neuen brasilianischen Umwelt 
angelegt wurde, bei spezifischen Bezeichnungen für Wagen und Karren (Charrete < pt. charrete, Arranja ,Wagen' (aus ,Spinne'), s. o.), dem Boden (Piso) oder dem in Europa zum Auswanderungszeitpunkt noch nicht stark etablierten Getreide Mais (Milje/Milgen < pt. milho). Im Bereich der Nahrungsmittel werden insbesondere südländische Früchte (Pêssego ,Pfirsich', Batat < pt. batata, evtl. über die Bezeichnung für Süßkartoffel, später verallgemeinert auf ,Kartoffel') und neue bzw. brasilianische Zubereitungsarten mit Lusitanismen ausgedrückt (Churrasco/Spetto ,Grillen's. o., Puffmilje/Bocksmilje ,Popcorn'; im Standarddt. als Anglizismus). Daneben spielt die brasilianische Tierwelt eine Rolle (Morcego „Fledermaus', Mosquito/Moskite ,Stechmücke', Mule ,Lasttier'). Auch Bereiche der Körperhygiene werden durch den Kontakt zum Portugiesischen geprägt, vgl. Bart (ab)mache ,rasieren' (s. o.), Sabonett/Sabonete ,Seife' < pt. sabonete). Daneben finden sich auch einige Bezeichnungen für Familienmitglieder, vgl. die expressiven Bezeichnungen Wowwo < vovô ,Großvater' und Wowwe < vovó ,Großmutter', daneben Cunhado ,Schwager'. Auch ein expressiver Ausdruck wie Lärm wird durch den Lusitanismus Barulje < barulho wiedergegeben. Schließlich sind auch technische und spielerische Neuerungen (der damaligen Zeit) durch Lusitanismen repräsentiert, vgl. Fosfo/Fosforo/Fosfore ,Streichholz', Isqueiro ,Anzünder', Biciclett ,Fahrrad' oder die Bezeichnungen für Spiel und Sport, etwa Bolle ,Ball' < bola sowie die Bezeichnungen für eine Schleuder: Bodock < bodoque, Funde < funda, Stelinge, estilingue'.

Fast in allen Fällen stehen die Lusitanismen bei den Spontanantworten in Konkurrenz zu deutschen Wörtern, die größtenteils sogar dominieren. In hoher Frequenz treten Lusitanismen besonders bei Assude ,Fischteich', Sabonett ,Seife', Milje ,Mais', den Tierbezeichnungen Moskite und Morcego sowie bei Spiel und Sport auf (Funde, Botock, Stelinge ,Schleuder', Bolle ,Ball', Biciclett ,Fahrrad'). Beim Großteil des hier erfragten Wortschatzes stehen also sowohl die deutsche als auch die entlehnte Variante zur Verfügung, das Vokabular ist durch hohe Variation geprägt.

\section{Fazit}

Der vorliegende Beitrag beschäftigt sich mit der lexikalischen Variation im Deutschen der Nachfahren böhmischer Einwanderer an drei Orten in Rio Grande do Sul, Brasilien. Auf Grundlage von Befragungsdaten wurde untersucht, wie sich das Lexikon entwickelt hat und welche Rolle dabei deutschsprachiger Dialektausgleich, innersprachlicher lexikalischer Ausbau und semantischer Wandel sowie der Kontakt zum Portugiesischen gespielt haben. Obwohl die Datenbasis überschaubar ist und sowohl um weitere Befragungsdaten als auch um eine Analyse freier gesprochener Daten ergänzt werden sollte, lassen sich einige Tendenzen erkennen:

a) Die Daten spiegeln historische Ausgleichsprozesse der deutschen Dialekte noch zu einem stärkeren Ausmaß als den Kontakt zum Portugiesischen. Dies hat einerseits mit der Erhebungsmethodik zu tun: Die Gewährspersonen sollten dezidiert Deutsch sprechen, sie wussten, dass es um eine Erhebung zu ihrer deutschen Alltagssprache geht und wurden von einer Gruppe hauptsächlich deutscher Akademiker/-innen befragt. Die 
Aufmerksamkeit war damit dezidiert auf das Deutsche gelenkt und die Gewährspersonen kontrollierten ihre Antworten entsprechend. Andererseits kann es aber auch die Entwicklung deutschsprachiger Varietäten in Brasilien widerspiegeln, die sich zunächst in der Einwanderungszeit v. a. im Kontakt unter Deutschsprachigen gestaltete (vgl. Altenhofen 2016 oder Auer 2005 für Rekonstruktionen der soziolinguistischen Prozesse) und erst mit der Zeit immer mehr auch durch den Kontakt mit dem Portugiesischen gestärkt wurde - am massivsten seit den 30er-Jahren des 20. Jahrhunderts mit wem Wegbrechen des deutschen Schulsystems und zunehmender Urbanisierung. Insbesondere die hohe Zahl an wmd. lexikalischen Einheiten zeigt, dass die hunsrückischen Varietäten als akrolektale Leitvarietäten angesehen wurden. Die kaum mehr zu findenden Spuren des nordböhmischen Ausgangsdialekts lassen auf die Übernahme immer mehr standard Varianten und wmd. Varianten schließen (vgl. auch die Ergebnisse von HABEL, 2017 und PREDIGER, 2019), wobei Agudo sich gegenüber den anderen Orten als standardnäher darstellt, indem einige westmd. Varianten dort nicht vorkommen.

b) Das deutschsprachige Lexikon wird in Brasilien um Neologismen erweitert, um den neuen Kontexten und den weiteren Veränderungen in Brasilien gerecht zu werden. Insbesondere tritt in unseren Daten die Komposition als häufig genutztes Wortbildungsmittel hervor, um Neologismen zu erzeugen. Hier ergibt sich eine Brücke zum deutsch-portugiesischen Sprachkontakt, indem zahlreiche Komposita hybrid aus deutschem und aus dem Portugiesischen entlehnten Material gebildet werden. Weiterhin konnte eine angepasste Nutzung deutscher Lexik mit Bezug auf die brasilianische Umgebung durch Bedeutungsveränderungen bei einigen Lexemen festgestellt werden (Bedeutungsverengung bei Spießbraten oder Rücken, -erweiterung bei Sirup, Gewitter, verschiebung bei Sumpf oder Flusse). Die Lexik wird also kontextsensitiv den Lebensbedingungen angepasst.

c) Der Kontakt zum Portugiesischen prägt die Lexik durch Entlehnungen. Es wurden hauptsächlich lexikalische Entlehnungen festgestellt, die zumeist Integrationsschritte auf lautlichem Niveau, v. a. geprägt durch Vokalreduktion in Nebensilben und durch Apokope, aufzeigten. Materialbedingt kamen kaum Lehnwortbildungen vor - dies wäre bei stärkerem Einbezug von Verben häufiger zu beobachten gewesen. Vor allem aber sind die Effekte auf lexikalischer Ebene angesiedelt. Die Daten deuten bei den befragten Gewährspersonen auf den Erhalt des Deutschen bei leichtem kulturellen Druck durch die portugiesische Umgebungssprache hin (vgl. die in Kap. 3 angesprochene Typologie von THOMASON/KAUFMAN 1988). Jedoch gilt es zu bedenken, dass über Generationen hinweg bei fehlender oder nur geringfügiger Weitergabe der deutschen Varietäten an Folgegenerationen ein kurzfristiger Sprachwechsel in vielen Familien nicht unwahrscheinlich ist.

d) Nur wenige Entlehnungen scheinen deutsche lexikalische Einheiten vollkommen abzulösen. Dass den Gewährspersonen nur ein Lusitanismus und kein deutsches Gegenstück in den Sinn kommt, ist fast nur bei Milje ,Mais' der Fall, wobei der Mais in Europa zur Auswanderungszeit keine große Rolle im Getreideanbau spielte und das Wort 
Mais daher eventuell schlicht unbekannt war. In den meisten Fällen dagegen ist den Gewährspersonen neben einem Lusitanismus auch eine deutsche Entsprechung bewusst, wie die Daten zeigen. Es wäre an einer gebrauchsbasierten Analyse, semantische oder stilistische Unterschiede bei der Nutzung von ererbten und entlehnten Wörtern zu untersuchen (vgl. etwa Gärtner 2011). In unserer Untersuchung lässt sich tendenziell ersehen, dass die Variation dadurch geprägt ist, dass Bereiche des Körpers, seiner Kleidung und bäuerlicher und handwerklicher Tätigkeiten sowie relevante Tiere und Nahrungsmittel des üblichen mitteleuropäischen Bauernhofs deutschsprachig ausgedrückt werden, ohne dass Lusitanismen hier überhaupt eine Rolle spielen. Dort, wo die bäuerliche Welt relativ ähnlich nach Südamerika transferiert wurde, ist die deutschsprachige Lexik offenbar am stabilsten. Dinge hingegen, die in der neuen Umgebung neu hinzutreten (Südfrüchte, Essenszubereitung), neu entwickelt werden (Techniken, Spiel und Sport) oder neu ausgelegt werden können (Fischteich, Wagentechnik etc.), werden am ehesten durch Lusitanismen bezeichnet.

Insgesamt zeigen die vorliegenden Analysen, dass die Sprache der böhmischen Einwander/-innen und ihrer Nachfahren eine komplexe Entwicklung in einem Varietäten- und Sprachenkomplex durchläuft, in dem akrolektale Wirkung sowohl innerhalb der deutschsprachigen Varietäten v. a von den wmd. geprägten Varietäten (Hunsrückisch) - mit Resultat in einem weitgehenden Sprachwechsel - und dem Standard-(Schrift-)Deutschen als auch im Sprachgeflecht vom Portugiesischen ausging und bis heute ausgeht. Die vorliegende Untersuchung kann nur einen Baustein in der Analyse dieser komplexen Verflechtungen leisten.

\section{Literaturverzeichnis}

ALTENHOFEN, Cléo V. Hunsrückisch in Rio Grande do Sul. Ein Beitrag zur Beschreibung einer deutschbrasilianischen Dialektvarietät im Kontakt mit dem Portugiesischen. Stuttgart: Steiner, 1996.

ALTENHOFEN, Cléo V . Standard und Substandard bei den Hunsrückern in Brasilien: Variation und Dachsprachenwechsel des Deutschen im Kontakt mit dem Portugiesischen. In: LENZ, Alexandra N.: German Abroad. Perspektiven der Variationslinguistik, Sprachkontakt- und Mehrsprachigkeitsforschung. Wien: Vienna University Press, 2016. S. 103-129. https://doi.org/10.14220/9783737005975.103

AUER, Peter. A(nother) scenario for new dialect formation. The German koine in Rio Grande do Sul (Brazil). In: MELANDER, Björn (Hg.): Språk i tid. Studier tillägnade Mats Thelander på 60-årsdagen. Uppsala: Uppsala Universitet, 2005. S. 57-70.

CHRISTILLINO, Christiano. Estranhos em seu próprio chão: o processo de apropriações de terras na Província de São Pedro do Rio Grande do Sul (o Vale do Taquari no período de 18401889). (Masterarbeit, Geschichte Lateinamerikas, UNISINOS). São Leopoldo: UNISINOS, 2004.

CUNHA, Jorge L. da. Imigração e colonização alemã. In: PICCOLO, Helga I. L.; PANDOIN, Maria M. (Orgs.). Passo Fundo: Méritos, 2006. S. 279-300. 
DtW = Deutsches Wörterbuch. Von Jacob und Wilhelm Grimm. 16 Bde. Leipzig: Hirzel, 18541961. Im Wörterbuchnetz: <http://woerterbuchnetz.de/cgi-bin/WBNetz/wbgui_py?sigle= DWB $>$, abgerufen am: 12.08.2020.

DWDS = Digitales Wörterbuch der deutschen Sprache. Hrsg. v. der Berlin-Brandenburgischen Akademie der Wissenschaften. <https://www.dwds.de/>, abgerufen am: 12.08.2020.

FENDRICH, Henrique. Lista de imigrantes boêmios. In: FENDRICH, Henrique. Genealogia Boêmia: boêmios brasileiros em busca de sua história. https://genealogiaboemia.wordpress.com/2016/01/13/lista-de-imigrantes-boemios-list-ofbohemian-immigrants-2/, abgerufen am: 11.08.2020.

FLORES, Hilda Agnes Hübner. Canção dos imigrantes. Porto Alegre: EST/EDUCS, 1983.

FLORES, Hilda Agnes Hübner. Imigrantes boêmios. Porto Alegre: Martins Livreiro, 2015.

GÄRTNER, Angelika. "Der schnodert em alemão"-Beispiele portugiesischer Entlehnungen und deutsch-portugiesischer Sprachmischungen aus Südbrasilien (São Bento do Sul, SC). Pandaemonium Germanicum, n. 18, S. 248-282, 2011.

HABEL, Jussara M. Das böhmische Deutsch: perda e coineização de variantes do alemão de imigrantes boêmios no Rio Grande do Sul. Masterarbeit (Master, Sprachwissenschaft, UFRGS). Porto Alegre, UFRGS, 2017.

HEISLER, Cláudio Affonso. Imigrantes boêmios no Vale do Sampaio. In: ARENDT, Isabel Cristina; WITT, Marcos Antônio (Hrsg.). História, cultura e memória. 180 anos de imigração alemã. São Leopoldo: OIKOS, 2005. S. 271-275.

LAROQUE, Luís F. et al. Imigrantes açorianos e seus descendentes no Vale do Taquari, Rio Grande do Sul: processo histórico envolvendo movimentações e práticas socioculturais. Ensino, Humanidades. Revista Signos, a. 37, n. 2. S. 104-123, 2016. https://doi.org/10.22410/issn.1983-0378.v37i2a2016.1103

MÜLLER, Josef. Rheinisches Wörterbuch. Band V: L-M. Berlin und Bonn: Fritz Klopp Verlag, 1941.

NAUMANN, Bernd. Deutsch und Deutsche in São Bento do Sul/Santa Catarina 1873 - 2003. In: Zur Landschaftsökologie der Baía da Babitonga. Ein brasilianisch-bairisches Modellprojekt. Erlangen: Universitätsbibliothek Erlangen, 2004. S. 111-119.

NÜBLING, Damaris; DAMMEL, Antje; DUKE, Janet; SZCZEPANIAK, Renata. Historische Sprachwissenschaft des Deutschen. Eine Einführung in die Prinzipien des Sprachwandels. Narr Studienbücher. 5. Auflage. Narr Francke Attempo Verlag: Tübingen, 2017.

PfW = Pfälzisches Wörterbuch. 6 Bde. und ein Beiheft. Wiesbaden/Stuttgart: Steiner, 19651998. Im Wörterbuchnetz: <http://woerterbuchnetz.de/cgi-bin/WBNetz/wbgui_py?sigle= PfWB $>$, abgerufen am: 12.08.2020.

PREDIGER, Angélica. Topodinâmica do alemão falado em comunidades de imigração do norte da Boêmia no Brasil. 2019. 321 f. Dissertação (Promotion, Sprachwissenschaft, UFRGS). Porto Alegre, UFRGS, 2019.

PREDIGER, Angélica; KÜRSCHNER, Sebastian. Die Dynamik des gesprochenen Deutschen bei Nachfahren böhmischer Auswanderer in Südbrasilien. In: FÖLDES, Csaba (Hrsg.): 
Kontaktvarietäten des Deutschen im Ausland. Tübingen: Narr (Beiträge zur Interkulturellen Germanistik - BIG). Im Druck.

RADTKE, Edgar; THUN, Harald (Hrsg.). Neue Wege der romanischen Geolinguistik: Akten des Symposiums zur empirischen Dialektologie. Kiel: Westensee-Verl., 1996.

RADTKE, Edgar; THUN, Harald (Hrsg.). Neue Wege der romanischen Geolinguistik: Eine Bilanz. In: RADTKE, Edgar; THUN, Harald (Hrsg.). Neue Wege der romanischen Geolinguistik: Akten des Symposiums zur empirischen Dialektologie. Kiel: Westensee-Verl., 1996. S. 1-24.

ROSENBERG, Peter. Lateinamerika. In: PLEWNIA, Albrecht; RIEHL, Claudia Maria (Hrsg.): Handbuch der deutschen Sprachminderheiten in Übersee. Tübingen: Narr, 2018. S. 193-264.

SCHMID, Hans Ulrich. Einführung in die deutsche Sprachgeschichte. Stuttgart: Metzler. 2009. https://doi.org/10.1007/978-3-476-05230-8

STAUB, Augostinus. O empréstimo lingüístico: um estudo de caso. Porto Alegre: Acadêmica, 1983.

STEDJE, Astrid. Deutsche Sprache gestern und heute. 6. Aufl. Paderborn: Fink. 2007.

TAVARES DE BARROS, Fernando Hélio. Topodinamica del Hunsrückisch: cartografia y ejemplos del proceso de cambio y manutencion del lexico en contexto de migracion. 2019. $309 \mathrm{f}$. Dissertação (Promotion, FB10 Sprach- und Literaturwissenschaften, Universität Bremen). 2019.

THOMASON, Sarah Gray; KAUFMAN, Terrence. Language contact, creolization, and genetic linguistics. Berkeley / Los Angeles: The University of California Press, 1988.

THUN, Harald. A dialetologia pluridimensional no Rio da Prata. In: ZILLES, Ana Maria Stahl (Hrsg.). Estudos de variação lingüística no Brasil e no Cone Sul. Porto Alegre: Ed. da UFRGS, 2005a. S. 63-92.

THUN, Harald. Variation im Gespräch zwischen Informant und Explorator. In: LENZ, Alexandra N.; MATTHEIER, Klaus J. (Hrsg.). Varietäten - Theorie und Empirie. Frankfurt a. Main [u. a.]: Lang, 2005b. S. 97-127.

THUN, Harald. A geolinguística pluridimensional, a história social e a história das línguas. In: AGUILERA, Vanderci de A. (Org.). Para a história do português brasileiro. Londrina: EDUAL, 2009. S. 533-558.

THUN, Harald. Pluridimensional Cartography. In: LAMELI, Alfred et al. Language mapping. Berlin: De Gruyter Mouton, 2011. S. 506-523. https://doi.org/10.1515/9783110219166.1.506 THUN, Harald. Variação na interação entre informante e entrevistador. Trad. Cléo Vilson Altenhofen / Filipe Neckel. Cadernos de Tradução, Porto Alegre, n. 40, 2017. S. 82-107.

WILDFEUER, Alfred. Sprachenkontakt, Mehrsprachigkeit und Sprachverlust: deutschböhmisch-bairische Minderheitensprachen in den USA und in Neuseeland. Coleção: Linguistik - Impulse und Tendenzen, v. 73. Berlin: de Gruyter, 2017. https://doi.org/10.1515/9783110552812 